Rel at i ve si zes of at ons observed in el ectron moment um densi t i es

\begin{tabular}{|c|c|}
\hline 著者 & KOGA Toshi kat su \\
\hline $\begin{array}{l}\text { j our nal or } \\
\text { publ i cat i on titl e }\end{array}$ & The j our nal of chem cal physi cs \\
\hline vol une & 116 \\
\hline number & 16 \\
\hline page $r$ ange & $6910-6913$ \\
\hline year & 2002-04 22 \\
\hline URL & ht t p: //hdl . handl e. net /10258/815 \\
\hline
\end{tabular}




\title{
Relative sizes of atoms observed in electron momentum densities
}

\author{
Toshikatsu Kogaa) \\ Department of Applied Chemistry, Muroran Institute of Technology, Muroran, Hokkaido 050-8585, Japan
}

(Received 3 January 2002; accepted 1 February 2002)

The radial electron momentum densities $I(p)$ of atoms are known to reveal several local maxima and minima. For the 103 atoms from $\mathrm{H}$ to $\mathrm{Lr}$ in their ground states, we report that the reciprocal momenta $1 / p_{\max }$ and $1 / p_{\min }$, where $p_{\max }$ and $p_{\min }$ are the locations of the maxima and minima in $I(p)$, respectively, have good linear correlations with the relative sizes $R$ of atoms, defined based on the spherically averaged densities $\rho(r)$ in position space. (c) 2002 American Institute of Physics.

[DOI: $10.1063 / 1.1463445]$

\section{INTRODUCTION} by

The radial electron momentum density $I(p)$ is defined

$$
I(p) \equiv \sum_{i=1}^{N}\left\langle\delta\left(p-\left|\mathbf{p}_{i}\right|\right)\right\rangle,
$$

where $\delta(x)$ is the one-dimensional Dirac delta function and the brackets \langle\rangle stand for the expectation value over an $N$-electron momentum wave function $\Phi\left(\mathbf{y}_{1}, \ldots, \mathbf{y}_{N}\right)$ with $\mathbf{y}_{i}$ $\equiv\left(\mathbf{p}_{i}, \sigma_{i}\right)$ being the combined momentum-spin coordinates of the $i$ th electron. The momentum density $I(p)$ represents the probability density function for the momentum magnitude $\left|\mathbf{p}_{i}\right|$ of any electrons $i$ to be $p$, and is normalized to $N$, the number of electrons. It is important that the density $I(p)$ is intimately related with experimental measurements: Within the impulse approximation, the isotropic Compton profile $J(q)$ is given ${ }^{1}$ in terms of $I(p)$ by

$$
J(q)=\frac{1}{2} \int_{|q|}^{\infty} d p p^{-1} I(p),
$$

which conversely implies $I(p)=-2 p[d J(p) / d p]$.

Moreover, many physical properties are connected ${ }^{1-6}$ to the momentum moments $\left\langle p^{n}\right\rangle$ defined by

$$
\left\langle p^{n}\right\rangle \equiv \int_{0}^{\infty} d p p^{n} I(p) .
$$

Examples are: $\left\langle p^{-1}\right\rangle$ is twice the Compton peak height $J(0)$, $\langle p\rangle / \pi$ is close $^{7}$ to the Slater-Dirac exchange energy, $\left\langle p^{2}\right\rangle$ is twice the nonrelativistic electronic kinetic energy, $\left\langle p^{3}\right\rangle$ is approximately proportional $^{7,8}$ to the initial value of the Patterson function in $\mathrm{x}$-ray crystallography, and $\left\langle p^{4}\right\rangle$ determines ${ }^{9}$ the Breit-Pauli mass-velocity correction in quasirelativistic quantum theory.

In addition to the above important characteristics, the momentum densities $I(p)$ of atoms are known ${ }^{10-14}$ to have several local maxima and minima. In the present article, we point out that for the 103 neutral atoms $\mathrm{H}$ through $\mathrm{Lr}$ in their ground states, the momenta $p_{\max }$ and $p_{\min }$, where the maxima and minima are observed in $I(p)$, are related with

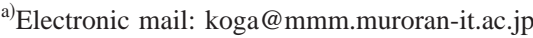

the relative sizes $R$ of atoms, which are derived ${ }^{15,16}$ from the analysis of the electron density $\rho(r)$ in position space. Hartree atomic units are used throughout.

\section{RELATIVE SIZES OF ATOMS}

Together with the additivity assumption for molecules and solids, various sizes were introduced ${ }^{17}$ empirically for atoms and ions, such as covalent, ionic, and metallic radii. To define intrinsic radii of atoms, before influenced by the molecular environment, Boyd ${ }^{15}$ examined several quantities derived from the spherically averaged density $\rho(r)$ in position space

$$
\rho(r) \equiv\left(4 \pi r^{2}\right)^{-1} \sum_{i=1}^{N}\left\langle\delta\left(r-\left|\mathbf{r}_{i}\right|\right)\right\rangle,
$$

and found that a density contour approach given by

$$
\rho(R)=C,
$$

yields the most appropriate result for the relative sizes $R$ of atoms, where $C$ is a constant common to all the atoms considered. Boyd used ${ }^{15} C=1 \times 10^{-4}$ for the 54 atoms from $\mathrm{H}$ (atomic number $Z=1)$ to $\mathrm{Xe}(Z=54)$, but a smaller value such as $C=5 \times 10^{-5}$ was suggested later ${ }^{16}$ when the 103 atoms $\mathrm{H}$ through $\operatorname{Lr}(Z=103)$ were examined. Since the calculated relative radii $R$ are substantially larger than the empirical radii due to the small value of $C$, scaling procedures were further introduced, ${ }^{15,16}$ which scale the theoretical radii $R$ down to values $\bar{R}$ with the magnitude of the empirical radii, referring to the univalent radii ${ }^{17}$ of Pauling for the first five rare-gas atoms. In the following discussion on the locations of the maxima and minima in the electron momentum density, however, we refer to the original radii $R$ obtained from the numerical Hartree-Fock densities ${ }^{18} \rho(r)$ with $C$ $=5 \times 10^{-5}$ as the relative sizes of atoms, since the two sets of values $R$ and $\bar{R}$ have parallel behavior against $Z$.

\section{LOCAL MAXIMA AND MINIMA IN RADIAL MOMENTUM DENSITIES}

For atomic systems, the presence of several local maxima and minima in the radial momentum densities $I(p)$ was reported in the literature, ${ }^{10-14}$ where we do not consider 
the two trivial local minima at $p=0$ and $\infty$. Among them, the study of Simas et al. ${ }^{14}$ which covers the 92 atoms from $\mathrm{H}$ to $\mathrm{U}$, appears to be most extensive. These authors clarified ${ }^{14}$ that the local maxima and minima in $I(p)$ are manifestation of outer electronic shells and intershell boundaries, respectively, in atoms.

Since all these studies are based on approximate Roothaan-Hartree-Fock wave functions, we have first reexamined the extremum characteristics of $I(p)$ using the numerical Hartree-Fock wave functions ${ }^{19}$ for the 103 atoms $\mathrm{H}$ through $\mathrm{Lr}$ in their experimental ground states. ${ }^{20,21}$ Fortunately, the previous observations remain unaltered. Based on the decomposition of $I(p)$ into shell contributions, we have also confirmed that the local maxima in $I(p)$ originate from outer electronic shells. The numbers of local maxima in the Hartree-Fock $I(p)$ of the 103 atoms are summarized as follows:

(i) Seven atoms with $Z=1,2,6-10$ have a single maximum;

(ii) 35 atoms with $Z=3-5,11-18,21-36,46,48-54$ have two local maxima;

(iii) 48 atoms with $Z=19,20,37-45,47,55-86,100-$ 103 have three local maxima;

(iv) 13 atoms with $Z=87-99$ have four local maxima.

Except for the case (i), there are local minima between two successive local maxima. Thus the number of local minima is less than the number of local maxima by one in every atom.

The correspondence between the local maxima in $I(p)$ and the outer electronic shells suggests that the locations $p_{\max }$ of these maxima would have some definite relations with the relative size $R$ of atoms, because the outer shells also give a significant contribution to the tail region of $\rho(r)$ from which $R$ is defined. The smallest (or first) $p_{\max }$, corresponding to the outermost shell, is our first candidate for the anticipated correlation. Moreover, we expect a positive correlation between the reciprocal momenta $1 / p_{\max }$ and the relative sizes $R$, since the position and momentum representations emphasize inverse regions of the respective spaces.

The correlation between the reciprocals of the first $p_{\max }$ and the relative sizes $R$ is plotted in Fig. 1(a) for the 103 atoms from $\mathrm{H}$ to Lr. We observe that $1 / p_{\max }$ and $R$ are positively correlated, but the relation is not clear when the 103 atoms are considered all together. In fact, the correlation coefficient (CC) is only 0.935 for a linear regression in the form of

$$
1 / p_{\max } \cong a R+b,
$$

where $a$ and $b$ are least square parameters. However, we find a good correlation between $1 / p_{\max }$ and $R$, if the 103 atoms are separated into three groups based on the numbers of electrons in the outermost $s$ and $p$ subshells; 16 atoms with $s^{1}$ configurations, 56 atoms with $s^{2}$ configurations, and 30 atoms with $s^{2} p^{k}(k=1-6)$ configurations. (We treated the Pd atom $(Z=46)$ as a sole exception, since it lies in the fifth period in spite of the vacant $O$ shell.) Figure 1(a) shows that there is a good linear correlation between $1 / p_{\max }$ and $R$ of the atoms in each group. The CCs in the regression analysis [Eq.
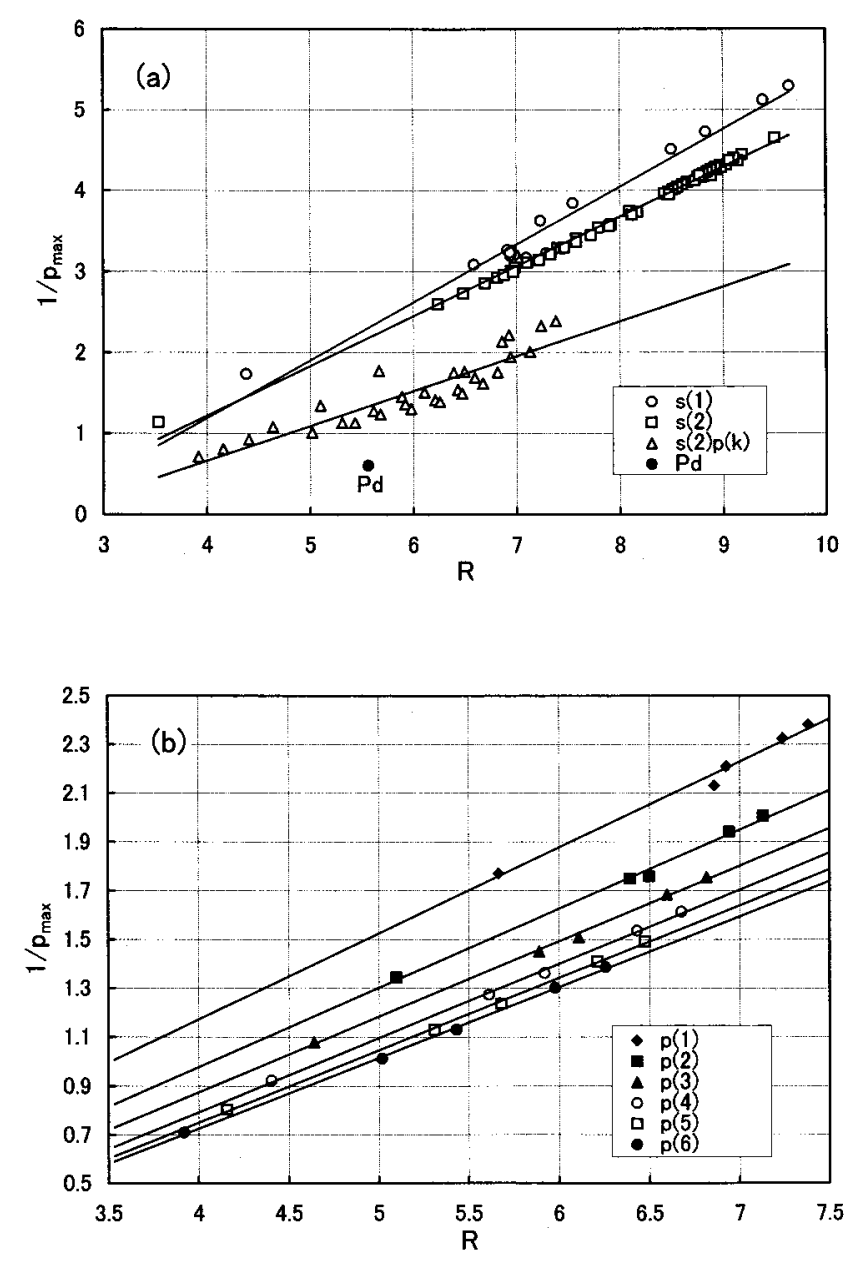

FIG. 1. Correlation between the reciprocals of the first $p_{\max }$ and the relative sizes $R$. (a) 103 atoms $\mathrm{H}$ through Lr. (b) 30 atoms with the outermost $s^{2} p^{k}$ ( $k=1-6)$ configurations.

(6)] are now $0.987,0.998$, and 0.913 for the three groups, respectively, as summarized in Table I. The poorest correlation for the atoms with $s^{2} p^{k}$ configurations is improved, when the group is further decomposed into six subgroups on the basis of the number $k$ of the $p$ electrons. The results are summarized in Fig. 1(b) and Table I; the CCs are never smaller than 0.993 . We thus conclude that the locations $p_{\max }$ of the first maxima in the electron momentum densities $I(p)$

TABLE I. Linear correlation between the reciprocal first $p_{\max }$ and $R$.

\begin{tabular}{|c|c|c|c|c|}
\hline $\begin{array}{l}\text { Outermost } s p \\
\text { configuration }\end{array}$ & $\begin{array}{l}\text { Number } \\
\text { of atoms }\end{array}$ & $a$ & $b$ & $\mathrm{CC}$ \\
\hline All & 103 & 0.810103 & -2.916880 & 0.935090 \\
\hline$s^{1}$ & 16 & 0.715001 & -1.674131 & 0.986557 \\
\hline$s^{2}$ & 56 & 0.615120 & -1.244998 & 0.997703 \\
\hline$s^{2} p^{k}(k=1-6)$ & $30^{\mathrm{a}}$ & 0.430253 & -1.062814 & 0.913300 \\
\hline$s^{2} p^{1}$ & 5 & 0.352493 & -0.237610 & 0.993484 \\
\hline$s^{2} p^{2}$ & 5 & 0.324216 & -0.319457 & 0.997717 \\
\hline$s^{2} p^{3}$ & 5 & 0.309095 & -0.361931 & 0.998951 \\
\hline$s^{2} p^{4}$ & 5 & 0.303583 & -0.421738 & 0.999257 \\
\hline$s^{2} p^{5}$ & 5 & 0.296491 & -0.436294 & 0.999427 \\
\hline$s^{2} p^{6}$ & $5^{\mathrm{a}}$ & 0.289660 & -0.433062 & 0.999521 \\
\hline
\end{tabular}

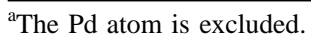



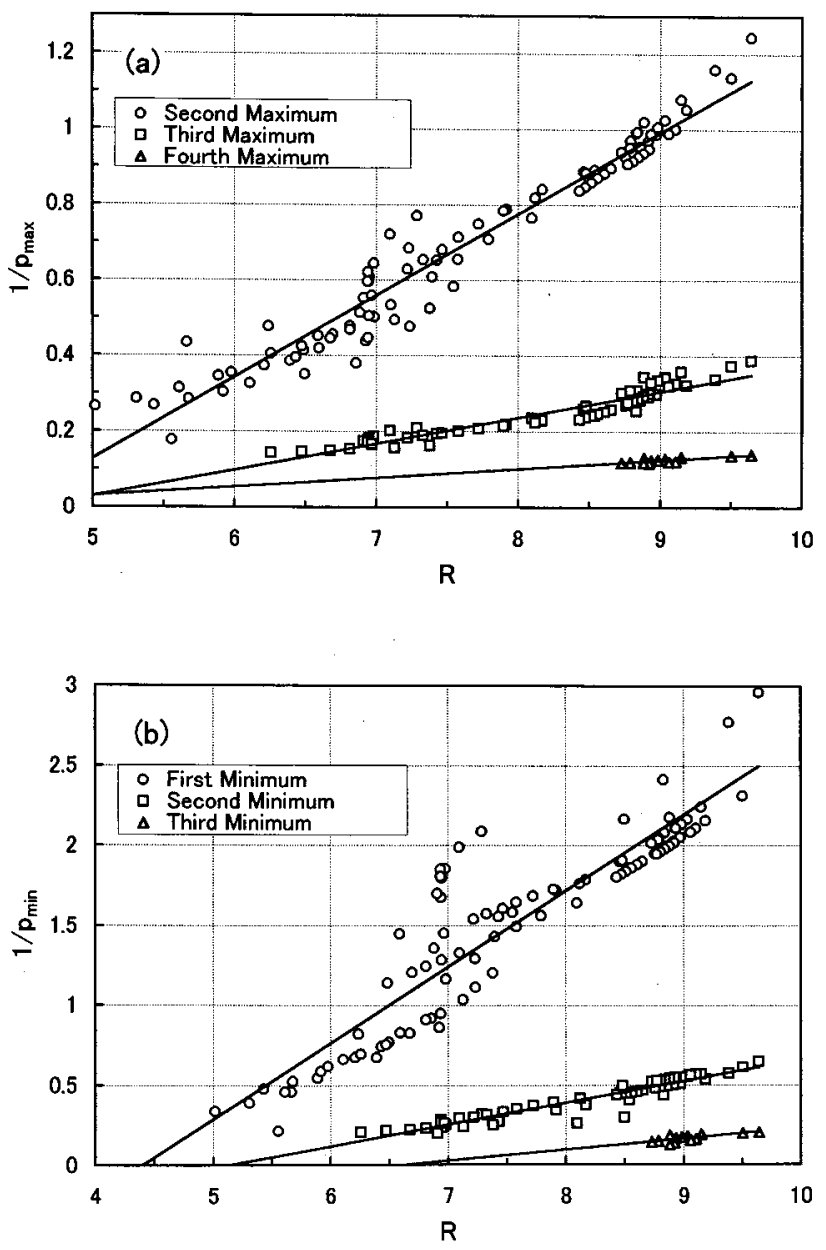

FIG. 2. Correlation between the reciprocals of $p_{\max }$ or $p_{\min }$ and the relative sizes $R$. (a) $1 / p_{\max }$ and $R$. (b) $1 / p_{\min }$ and $R$.

are connected with the relative sizes $R$ of atoms, if the difference in the numbers of the outermost $s$ and $p$ electrons is taken into account.

The classification of the $1 / p_{\max }-R$ correlation based on the outermost $s p$ configurations can be rationalized as follows. The numerical values of the first $p_{\max }$ are smaller than unity in most atoms, and are governed by the momentum densities $I(p)$ in their small $p$ regions. Moreover, the small$p$ asymptotic behavior of the subshell momentum density $I_{n l}(p)$, specified by the principal $n$ and azimuthal $l$ quantum numbers, is known ${ }^{19}$ to be

$$
I_{n l}(p)=A_{n l} p^{2 l+2}+B_{n l} p^{2 l+4}+O\left(p^{2 l+6}\right),
$$

where $A_{n l}$ and $B_{n l}$ are constants, which include the (2l)th and $(2 l+2)$ th derivatives at $p=0$ of the spherically averaged density $\Pi_{n l}(p)=I_{n l}(p) /\left(4 \pi p^{2}\right)$, respectively. Equation (7) clarifies that the leading $p^{2}$ and $p^{4}$ terms, arising from the outermost $s(l=0)$ and $p(l=1)$ subshells, give the predominant contribution to the value of the first $p_{\max }$.

Above the correlation between the reciprocal momenta $1 / p_{\max }$ of the first maxima in $I(p)$ and the relative sizes $R$ has been discussed. We have also examined the possibility of the correlation between $R$ and $1 / p_{\max }$ of the second, third, and fourth maxima, though all the 103 atoms do not always
TABLE II. Linear correlations between $1 / p_{\max }$ and $R$ and between $1 / p_{\min }$ and $R$.

\begin{tabular}{rcccc}
\hline \hline Extremum & $\begin{array}{c}\text { Number } \\
\text { of atoms }\end{array}$ & $a$ & $b$ & CC \\
\hline Maximum & & & & \\
First & 103 & 0.810103 & -2.916880 & 0.935090 \\
Second & 96 & 0.216273 & -0.954456 & 0.974050 \\
Third & 61 & 0.068905 & -0.315087 & 0.944805 \\
Fourth & 13 & 0.023519 & -0.087281 & 0.831485 \\
Minimum & & & & \\
First & 96 & 0.476119 & -2.092869 & 0.917366 \\
Second & 61 & 0.135641 & -0.696451 & 0.950843 \\
Third & 13 & 0.068291 & -0.453771 & 0.742036 \\
\hline \hline
\end{tabular}

have these maxima. A positive linear correlation is again observed as summarized in Fig. 2(a) and Table II. For the second and third maxima in $I(p)$, the CCs $(0.974$ and 0.945 , respectively) are larger than the first, and the reciprocal momenta of these maxima, due to the electrons in the second and third outermost shells, also reflect the relative sizes of atoms. However, the correlation is poorer for the fourth maxima, as the $\mathrm{CC}$ value $(0.831)$ shows.

From the observed correlation between $1 / p_{\max }$ and $R$, we further anticipate a positive correlation between $1 / p_{\min }$ and $R$

$$
1 / p_{\min } \cong a R+b,
$$

where $p_{\min }$ is the locations of the minima in the momentum densities $I(p)$. As plotted in Fig. 2(b), our anticipation is found to be correct. The values of CCs given in Table II show that for the first and second minima, the degree of correlation is analogous to that of the maxima, but it is poor for the third minimum.

\section{SUMMARY}

For the 103 atoms from $\mathrm{H}$ to $\mathrm{Lr}$ in their ground states, we have shown that the reciprocal momenta $1 / p_{\max }$ and $1 / p_{\min }$ have good linear correlations with the relative sizes $R$ of atoms, where $p_{\max }$ and $p_{\min }$ are the locations of the maxima and minima in the radial electron momentum densities $I(p)$, respectively.

\section{ACKNOWLEDGMENT}

This work was supported in part by a Grant-in-Aid for Scientific Research from the Ministry of Education of Japan.

${ }^{1}$ B. G. Williams, Compton Scattering: The Investigation of Electron Momentum Distributions (McGraw-Hill, New York, 1977).

${ }^{2}$ R. Benesch and V. H. Smith, Jr., in Wave Mechanics-The First Fifty Years, edited by W. C. Price, S. S. Chissick, and T. Ravensdale (Butterworths, London, 1973), pp. 357-377.

${ }^{3}$ L. Mendelsohn and V. H. Smith, Jr., in Compton Scattering: The Investigation of Electron Momentum Distributions, edited by B. G. Williams (McGraw-Hill, New York, 1977), pp. 102-138.

${ }^{4}$ P. Kaijser and V. H. Smith, Jr., Adv. Quantum Chem. 10, 37 (1977).

${ }^{5}$ W. M. Westgate, R. P. Sagar, A. Farazdel, V. H. Smith, Jr., A. M. Simas, and A. J. Thakkar, At. Data Nucl. Data Tables 48, 213 (1991).

${ }^{6}$ T. Koga and A. J. Thakkar, J. Phys. B 29, 2973 (1996).

${ }^{7}$ R. K. Pathak, B. S. Sharma, and A. J. Thakkar, J. Chem. Phys. 85, 958 (1986).

${ }^{8}$ A. J. Thakkar and W. A. Pedersen, Int. J. Quantum Chem. Symp. 24, 327 (1990). 
${ }^{9}$ H. A. Bethe and E. E. Salpeter, Quantum Mechanics of One- and TwoElectron Atoms (Plenum, New York, 1977), p. 170.

${ }^{10}$ W. E. Duncanson and C. A. Coulson, Proc. Phys. Soc. London 57, 190 (1945).

${ }^{11}$ W. E. Duncanson and C. A. Coulson, Proc. Phys. Soc. London 60, 175 (1948).

${ }^{12}$ W. Weyrich, P. Pattison, and B. G. Williams, Chem. Phys. 41, 271 (1979).

${ }^{13}$ S. R. Gadre, S. Chakravorty, and R. K. Pathak, J. Chem. Phys. 78, 4581 (1983).

${ }^{14}$ A. M. Simas, W. M. Westgate, and V. H. Smith, Jr., J. Chem. Phys. 80, 2636 (1984).

${ }^{15}$ R. J. Boyd, J. Phys. B 10, 2283 (1977).
${ }^{16}$ T. Koga, J. Chem. Phys. 112, 6966 (2000).

${ }^{17}$ L. Pauling, The Nature of the Chemical Bond, 3rd ed. (Cornell University Press, Ithaca, 1960).

${ }^{18}$ T. Koga, Theor. Chim. Acta 95, 113 (1997).

${ }^{19}$ T. Koga, H. Matsuyama, H. Inomata, E. Romera, J. S. Dehesa, and A. J. Thakkar, J. Chem. Phys. 109, 1601 (1998).

${ }^{20}$ C. E. Moore, Ionization Potentials and Ionization Limits Derived from the Analysis of Optical Spectra. NSRDS-NBS 34 (Nat. Bur. Stand. U.S., Washington, DC, 1970).

${ }^{21}$ H. L. Anderson, A Physicist's Desk Reference (AIP, New York, 1989), p. 94. 